\title{
Retinal vascular tortuosity in obstructive sleep apnea
}

This article was published in the following Dove Press journal:

Clinical Ophthalmology

24 April 2013

Number of times this article has been viewed

\begin{abstract}
Amir Mohsenin'
Vahid Mohsenin ${ }^{2}$

Ron A Adelman'

'Department of Ophthalmology and Visual Science, Yale University School of Medicine, New Haven, Connecticut, USA; ${ }^{2}$ Yale Center for Sleep Medicine, Department of Medicine, Yale University School of Medicine, New Haven, Connecticut, USA
\end{abstract}

Correspondence: Ron A Adelman 40 Temple Street, New Haven, CT 065I0, USA

Tel +I 2037852020

Fax +I 2037855909

Email ron.adelman@yale.edu
Purpose: Endothelial dysfunction and vascular disease are common in obstructive sleep apnea (OSA). We sought to examine the retinal vascular manifestations of OSA.

Methods: Nine consecutive patients with OSA underwent ophthalmic examination regardless of any ocular complaints. Seven patients without OSA matched for demographics were used as controls. Fundus photographs from both eyes were used to quantitate retinal vascular tortuosity of the temporal arterial and venous arcades using ImageJ digital analysis software. The tortuosity of each vessel from the optic disc rim to the crossing point of a 5 disc diameter (5DD) circle and 10 disc diameter (10DD) circle centered on the optic disc were quantitated.

Results: The mean age of patients with OSA in the study was 52 years \pm SD of 10 years and 67 years \pm SD of 10 years in the control group. The apnea-hypopnea index in patients with OSA ranged from 12 to 102 events/hr of sleep. The nadir oxyhemoglobin saturation during sleep in patients with OSA ranged from $60 \%$ to $87 \%$. There was no significant difference in the frequency of diabetes or hypertension between the groups. Total tortuosity was increased at the 5DD $(P=0.011)$ and 10DD $(P=0.004)$ marks. Arterial tortuosity was significantly increased at the 10DD mark $(P=0.016)$. Venular tortuosity was increased at both the 5DD $(P=0.001)$ and 10DD $(P=0.028)$ marks.

Conclusion: Patients with OSA have increased retinal vascular tortuosity as compared to matched controls. Increased tortuosity of the retinal vasculature may be a novel association with OSA. A larger prospective study will be necessary to further explore this relationship and its clinical significance.

Keywords: retinal vessel tortuosity, sleep apnea, retinal vasculature, retinal vascular tortuosity

Obstructive sleep apnea (OSA) is a disorder characterized by repeated episodes of partial or complete closure of the upper airway during sleep. Airway closure results in respiratory effort-related arousals and fragmented sleep that cause daytime symptoms such as sleepiness, fatigue, and decreased concentration. During episodes of apnea, patients become hypoxemic and undergo arterial blood pressure surges and increased intracranial pressure. ${ }^{1}$ Current evidence suggests that inflammatory processes, oxidative stress and endothelial dysfunction may play roles in the pathogenesis of vascular complications in OSA. ${ }^{2}$ By itself and irrespective of common comorbid conditions like hypertension, OSA has been shown to be an independent risk factor for coronary artery disease, stroke and death. ${ }^{3,4}$ With the increasing prevalence of OSA and its implications for increased morbidity and mortality further research into ophthalmic associations is important.

Several ophthalmic conditions have been thus far linked to OSA including glaucoma, floppy eyelid syndrome, nonarteritic anterior ischemic optic neuropathy (NAION), optic 
disc edema, and diabetic retinopathy severity. ${ }^{5-8}$ While no definitive mechanism for ophthalmic associations has been elucidated, the alterations in blood pressure and hypoxemia seen in patients with OSA have been proposed as possible etiologies for glaucoma, NAION, optic disc edema, and the degree of diabetic retinopathy. More recent work by Glacet-Bernard et al. examined the association between OSA and retinal vein occlusion. ${ }^{9}$ They found a higher than expected prevalence of OSA in patients with vein occlusion proposing the idea that OSA may be a risk factor for vein occlusion.

Increased tortuosity of the retinal vasculature has been associated with diabetes, hypoxia, older age, hypertension, and elevated body mass index (BMI). ${ }^{10,11}$ As all of these aforementioned factors are also associated with OSA we hypothesized that patients with OSA will have alterations in their retinal vascular pattern. Our findings in this pilot study demonstrate increased retinal vascular tortuosity in OSA that appears to be independent of comorbid conditions like hypertension and diabetes.

\section{Methods}

Patients with OSA evaluated within the Yale Medical Group (New Haven, CT, USA) were consecutively asked to undergo ophthalmic examination regardless of any visual or eye complaints. These patients had undergone polysomnography according to the method endorsed by the American Academy of Sleep Medicine. Nine patients with OSA were enrolled into the study. Seven control subjects without OSA and matched for demographics were identified from patients within the Yale Medical Group undergoing complete eye examination and fundus photography for other reasons.

Basic demographic information, past medical history, and past ocular history along with ophthalmic data including visual acuity and intraocular pressure (IOP) were recorded. Inclusion criteria include the diagnosis of OSA and having had a complete dilated ophthalmic examination with fundus photography. Exclusion criteria were patients with central sleep apnea. Each patient had undergone a complete eye examination including visual acuity testing, IOP measurement, and slit lamp biomicroscopy with dilation and fundus photography. Fundus photographs were obtained with a Topcon fundus camera (Topcon, Oakland, NJ, USA). ImageJ software (National Institutes of Health, Bethesda, MD, USA) was used to obtain measurements of the temporal retinal arcades. Two concentric circles, one with a diameter of 5 disc diameters (5DD) and another with a diameter of 10 disc diameters (10DD) were drawn centered on the optic disc (Figure 1). Individual measurements of the superior

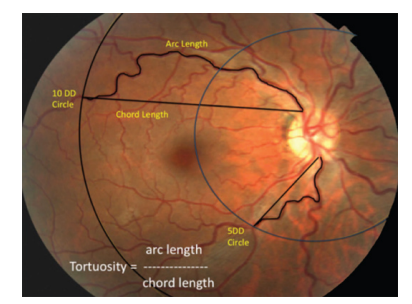

Figure I Measurement of tortuosity.

Notes: Two concentric circles with diameters of 5 disc diameters (5DD) and 10 disc diameters (IODD) were drawn centered on the optic disc. NIH ImageJ software was used to measure the arc and chord lengths of vessel segments as shown. Tortuosity was calculated by dividing the arc length by the chord length of each vessel segment.

and inferior arterial and venular arcades were obtained starting from the optic disc margin to the crossing point of the 5DD and 10DD circles. The arc and chord lengths of each vessel segment were obtained at the 5DD and 10DD points. Tortuosity was calculated by dividing the arc length by the chord length at each point as per previously published methods. ${ }^{12,13}$

Statistical analysis was performed with GraphPad Prism software (La Jolla, CA, USA). Data presented in graphs are expressed as mean $\pm \mathrm{SE}$. Otherwise the data is expressed as mean \pm SD throughout. Data were analyzed using Mann-Whitney non-parametric two-tailed tests for comparisons of the groups. Institutional Review Board approval from the Yale University Human Investigations Committee was obtained for this study.

\section{Results}

Table 1 summarizes the patient characteristics. The study included 9 patients with OSA and 7 control subjects. All patients in the OSA group were male while 6 out of 7 in the control group were male. Visual acuity and IOP were similar between groups. There were no significant differences in the frequency of hypertension or diabetes between the groups. Neither group had any documented vein occlusion, papilledema, ischemic optic neuropathy, or floppy eyelid syndrome. Of the 9 patients

Table I Patient demographics

\begin{tabular}{lll}
\hline & $\begin{array}{l}\text { Sleep apnea } \\
\mathbf{n = 9}\end{array}$ & $\begin{array}{l}\text { Controls } \\
\mathbf{n = 7}\end{array}$ \\
\hline Age (SD) & $52(10)$ & $67(10)$ \\
Sex & 9 males & 6 males, I female \\
LogMAR vision (SD) & $0.15(0.13)$ & $0.17(0.18)$ \\
Intraocular pressure (SD) & $19(4)$ & $18(6)$ \\
Hypertension & $7 / 9(78 \%)$ & $7 / 7(100 \%)$ \\
Diabetes mellitus & $2 / 9(22 \%)$ & $2 / 7(29 \%)$ \\
Glaucoma & $2 / 9(22 \%)$ & $3 / 7(43 \%)$ \\
\hline
\end{tabular}

Abbreviation: SD, standard deviation. 
with OSA in our study, 6 were on positive airway pressure treatment. The apnea-hypopnea index (AHI) in the patients with OSA ranged from 12 to 102 events/hr of sleep and the nadir oxyhemoglobin saturation during sleep in these patients ranged from $60 \%$ to $87 \%$. Examples of fundus photographs from the OSA group demonstrating increased retinal vasculature tortuosity are depicted in Figure 2.

\section{Arterial tortuosity}

Figure 3 represents the tortuosity averages of the superior and inferior arterial arcades at the 5DD and 10DD marks. Results demonstrate that patients with OSA $(1.226 \pm 0.107,95 \%$ confidence interval $[\mathrm{CI}][1.128,1.325])$ have a statistically significant $(P=0.016)$ increase in tortuosity measured at the 10DD as compared to controls $(1.067 \pm 0.019$, $95 \%$ CI $[1.02,1.113])$. Figure 4 represents subgroup analysis of the superior and inferior arterial arcades. At the 10DD mark, patients with OSA $(1.232 \pm 0.12,95 \%$ CI [1.121, 1.343]) have a significant $(P=0.01)$ increase in tortuosity of the superior arterial arcade as compared to controls $(1.08 \pm 0.03,95 \%$ CI $[1.035,1.134])$. Similarly at the 10DD mark, patients with OSA $(1.193 \pm 0.1,95 \%$ CI $[1.115,1.27])$ have a significant increase $(P=0.009)$ in tortuosity of the inferior arterial arcade as compared to controls (1.042 \pm 0.01 , 95\% CI [1.016, 1.069]).

\section{Venular tortuosity}

Figure 5 represents the tortuosity averages of the superior and inferior venous arcades at the 5DD and 10DD marks. At the 10DD mark, patients with OSA $(1.255 \pm 0.164,95 \%$ CI $[1.118,1.392])$ have a significant $(P=0.028)$ as compared to controls $(1.104 \pm 0.045,95 \%$ CI $[1.033,1.175])$. Similarly, at the 5DD mark, patients with OSA $(1.179 \pm 0.163,95 \%$ CI $[1.053,1.304])$ displayed a significant $(P=0.001)$ increase in venular tortuosity as compared to controls $(1.047 \pm 0.025,95 \%$ CI [1.024, 1.07]).

Subgroup analysis of superior and inferior venular arcade tortuosity is demonstrated in Figure 6. At the 10DD mark, patients with OSA $(1.267 \pm 0.177,95 \%$ CI $[1.12$, 1.415]) had a statistically significant $(P=0.003)$ increase in venular tortuosity of the superior arcade as compared to controls $(1.081 \pm 0.033,95 \%$ CI [1.041, 1.122]). There was no significant difference in tortuosity in the inferior venular arcades at 10DD. At 5DD, patients with OSA have a significant increase in venular tortuosity of both the superior $(P=0.001,1.188 \pm 0.184,95 \%$ CI $[1.046,1.329])$ and inferior $(P=0.011,1.17 \pm 0.149,95 \%$ CI $[1.056,1.285]$ arcades as compared to controls (superior: $1.041 \pm 0.025$, 95\% CI $[1.019,1.064])$, (inferior: $1.052 \pm 0.031,95 \%$ CI $[1.023,1.081])$.

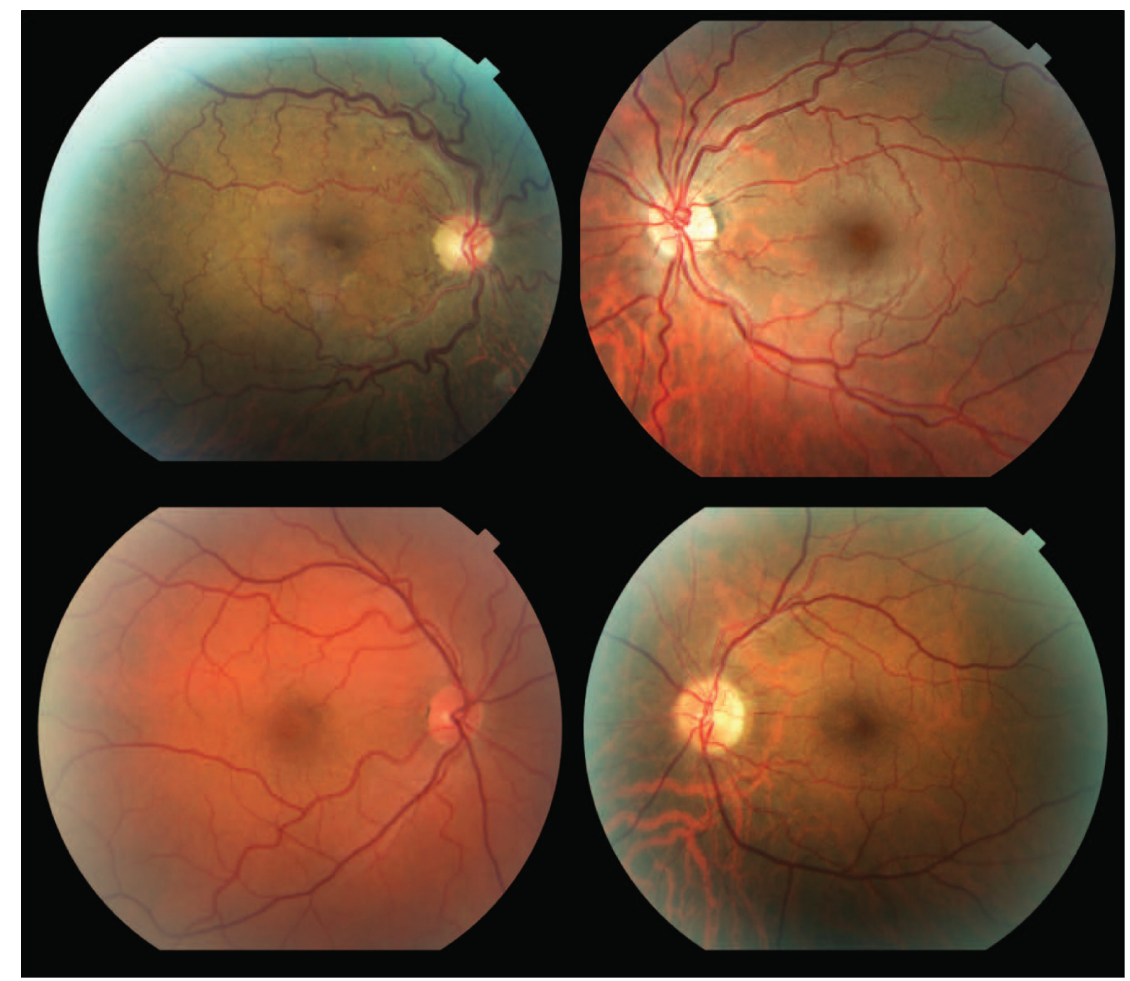

Figure 2 Representative fundus photographs from patients with OSA demonstrating increased tortuosity of the retinal vasculature. 


\section{Total arterial tortuosity}

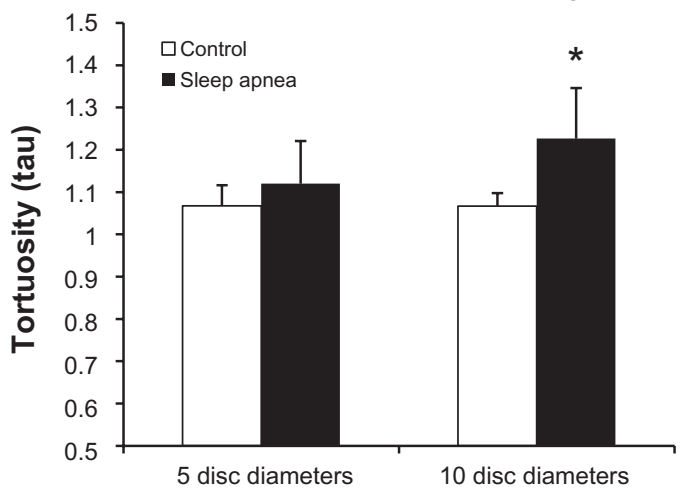

Figure 3 Total arterial tortuosity is significantly increased at the IODD mark $(P=0.016)$.

\section{Total tortuosity}

Figure 7 shows the combined tortuosity of both the arterial and venular arcades at each mark. At the 5DD mark, patients with OSA had a significant $(P=0.011)$ increase in total tortuosity $(1.149 \pm 0.131,95 \% \mathrm{CI}[1.049,1.25])$ as compared to controls (1.057 $\pm 0.024,95 \% \mathrm{CI}[1.035,1.079])$. Similarly, at the 10DD mark, patients with OSA had a significant

A

Superior arterial arcade

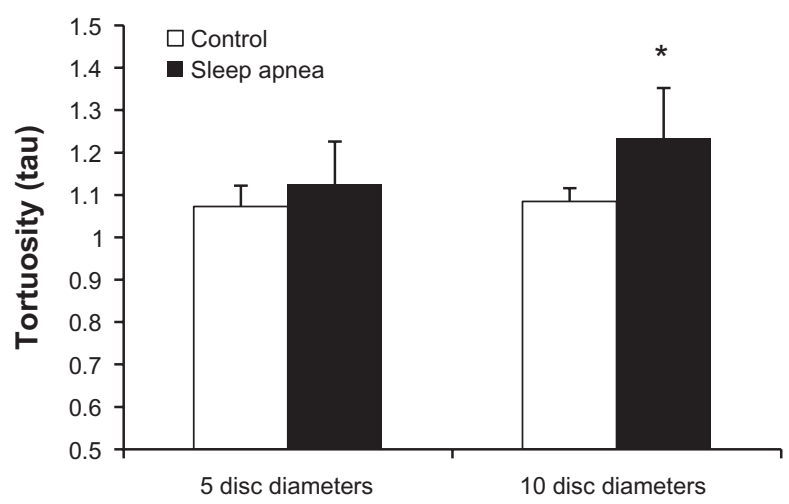

B

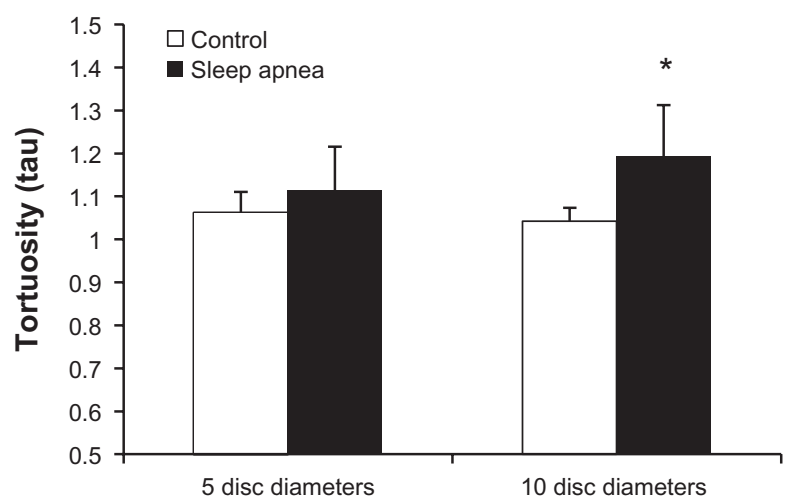

Figure 4 There is increased tortuosity of both the retinal $(\mathbf{A})$ superior $(P=0.0 \mathrm{I})$ and $(\mathbf{B})$ inferior $(P=0.009)$, arterial arcades at the IODD mark $(*=P<0.05)$.
Total venular tortuosity

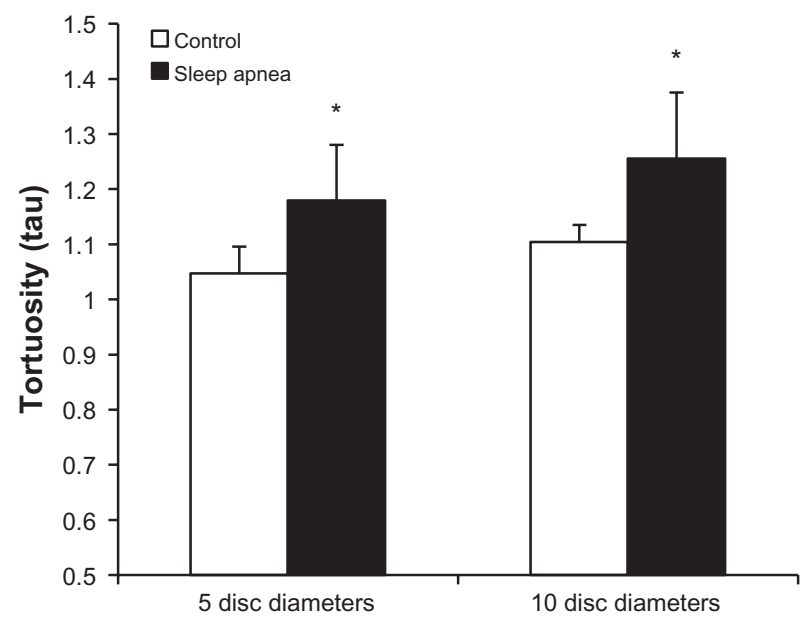

Figure 5 Total venular tortuosity is increased at both the 5DD $(P=0.001)$ and IODD $(P=0.028)$ marks $*=P<0.05$.

$(P=0.004)$ increase in total tortuosity $(1.223 \pm 0.127,95 \%$ CI $[1.125,1.321])$ as compared to controls $(1.091 \pm 0.04$, $95 \%$ CI $[1.041,1.141])$.

\section{Discussion}

Sleep-disordered breathing, of which OSA is the most common, is estimated to affect up to $24 \%$ of men and $9 \%$ of women in the United States. ${ }^{14}$ In addition, there are a projected 18 million believed to be untreated. ${ }^{15}$ As the prevalence of obesity continues to rise, more and more patients will be at risk. OSA is associated with endothelial dysfunction, systemic vasculopathy, and is an independent risk factor for increased cardiovascular morbidity and mortality making it imperative that patients be diagnosed and treated expeditiously.

Floppy eyelid syndrome, primary open angle glaucoma, normal tension glaucoma, NAION, optic disc edema and the degree of diabetic retinopathy have been all been linked to OSA. ${ }^{5-8}$ The majority of ophthalmic associations in OSA have potential vascular etiologies. During apneas, transient hypotension and hypoxemia may occur with repeated oxyhemoglobin desaturations as low as $30 \%$. This hypotension and hypoxemia has been proposed as a possible etiologic factor for glaucoma and NAION secondary to ischemic damage to the optic nerve. Optic disc edema may be secondary to the catecholamine release seen at termination of the apnea which results in blood pressure surges and increased intracranial pressure. Boland et al, examined the retina in patients with OSA looking for microvascular abnormalities including microaneurysms, hemorrhages, exudates, macular edema, intraretinal microvascular abnormalities, venous 
A

Superior venular arcade

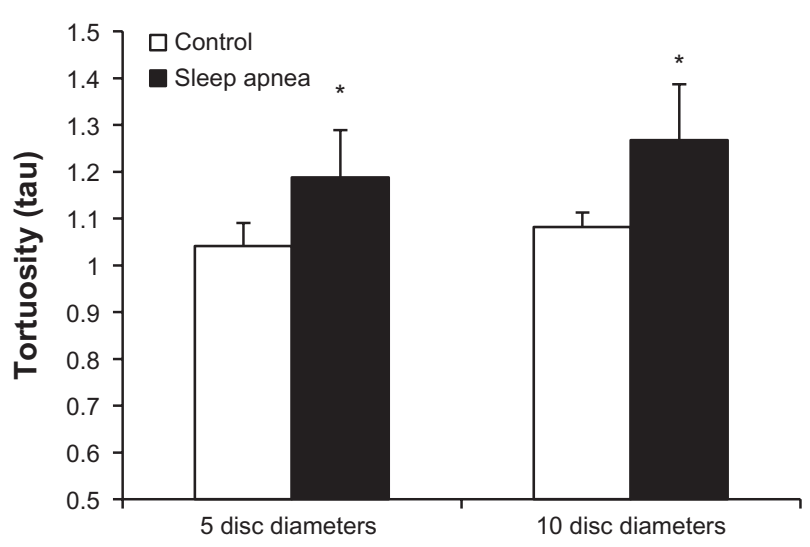

B

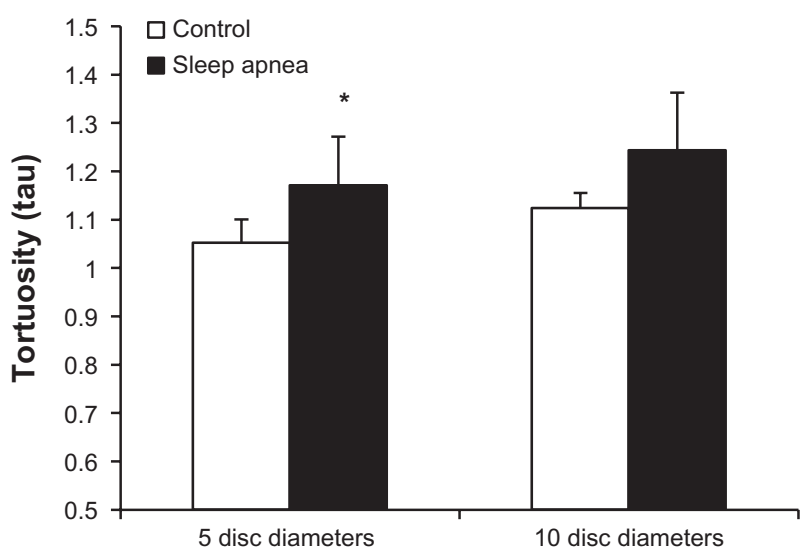

Figure 6 Tortuosity is significantly increased in the superior arcades at both the 5DD $(P=0.00 \mathrm{I})$ and IODD $(P=0.003)$ marks.

Note: The inferior venular arcade shows significant increased tortuosity at the 5DD mark only $(P=0.01 \mathrm{I})(*=P<0.05)$.

beading, neovascularization, and vitreous hemorrhage. ${ }^{16}$ Their study concluded that there was no notable relationship between sleep-disordered breathing and retinal abnormalities. In patients with both OSA and diabetes, the AHI and level of oxyhemoglobin desaturation have been shown to correlate with the degree of diabetic retinopathy. ${ }^{17}$ To our knowledge, the status of retinal vascular tortuosity has not yet been reported in OSA.

In our series, patients with OSA demonstrate increased retinal vascular tortuosity as compared to controls. The venous system in OSA was significantly more tortuous at both the 5DD and 10DD marks while the arterial system was more tortuous at the 10DD mark. As both diabetes and hypertension are associated with increased retinal vascular tortuosity we attempted to match the control group for these comorbidities. ${ }^{10}$ In our small cohort of patients, the increased tortuosity in OSA appears to be independent of the presence of hypertension or diabetes.
Total tortuosity

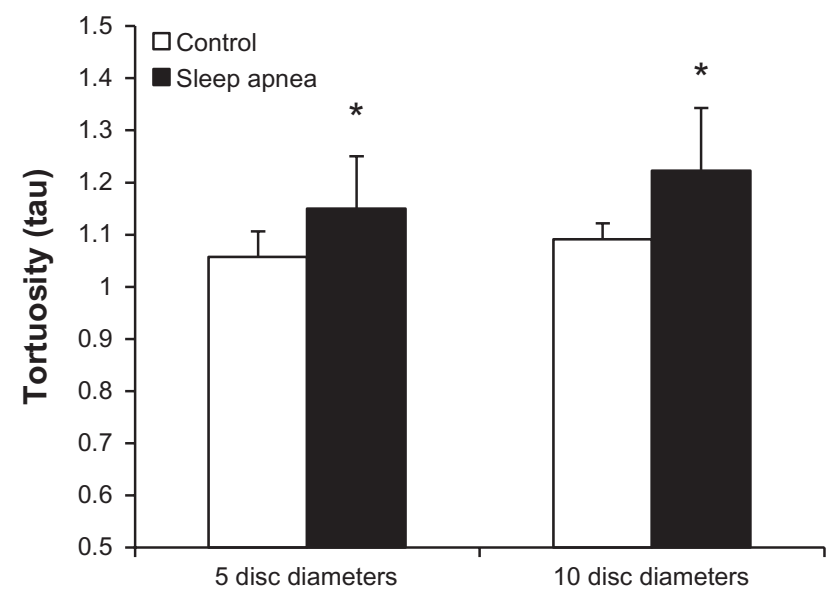

Figure 7 Tortuosity of the arteries and veins taken as a whole shows significantly increased tortuosity at 5DD $(P=0.01 \mathrm{I})$ and IODD $(P=0.004)$ in patients with obstructive sleep apnea $(*=P<0.05)$.

The mechanism of increased retinal vascular tortuosity in OSA is likely multi-factorial. Apneas result in significant intermittent arterial blood pressure surges, increased venular pressure, and increased intracranial pressure which can potentially lead to tortuosity due to increased shear wall stress. ${ }^{18}$ In addition, patients with OSA may have hypercapnia which has been shown to be associated with increased retinal arteriolar and capillary blood flow. ${ }^{19}$ The alteration of retinal blood flow may also be related to impaired cerebral autoregulation in OSA making ophthalmic vasculature more susceptible to shear stress during arterial and venous pressure swings during apneas. ${ }^{20} \mathrm{We}$ hypothesize that hypercapnia, blood pressure surges, and disrupted cerebral autoregulation may result in increased blood flow or blood volume in the retinal vasculature causing tortuosity.

While the significance of increased tortuosity is not yet clear, this finding is interesting in light of the recent associations made between vein occlusion and OSA. ${ }^{9}$ It is possible that increased tortuosity may result in turbulence or stagnation of blood flow predisposing these patients to retinal occlusive disease. There is also evidence that OSA is associated with a hypercoagulable state which would explain both the increased risk of cerebral stroke and retinal vein occlusion. ${ }^{21}$

There are several limitations to this study. First, this study is limited by the small number of patients in both the control and study groups. Second, the influence of hypertension or diabetes on our findings cannot be totally excluded. However, due to the comparable frequency of these two comorbidities, it is unlikely that they have a major effect on the difference in observed retinal vascular tortuosity. Third, we cannot exclude 
the effect of positive airway pressure treatment or obesity on retinal vascular changes. Finally, the severity of sleep apnea with respect to the apnea-hypopnea index and oxyhemoglobin saturations during sleep are important factors to evaluate in future studies. Although our study size is small, the patients with OSA demonstrated a wide range in the AHI and nadir oxyhemoglobin saturations showing that patients with both mild and severe sleep apnea displayed increased tortuosity.

In conclusion, patients with OSA have increased retinal vascular tortuosity. These retinal vascular changes may be related to the underlying systemic inflammatory state and alterations in cerebral hemodynamics seen in OSA. A prospective study with a larger sample size will be necessary to further explore this relationship and its clinical significance.

\section{Acknowledgment}

This work was partially supported by grants from the Newman's Own Foundation and Leir Foundation.

\section{Disclosure}

The authors report no conflicts of interest in this work.

\section{References}

1. Marrone O, Riccobono L, Salvaggio A, et al. Catecholamines and blood pressure in obstructive sleep apnea syndrome. Chest. 1993;103(3): $722-727$.

2. Jelic S, Padeletti M, Kawut SM, et al. Inflammation, oxidative stress, and repair capacity of the vascular endothelium in obstructive sleep apnea. Circulation. 2008;117(17):2270-2278.

3. Shah NA, Yaggi HK, Concato J, et al. Obstructive sleep apnea as a risk factor for coronary events or cardiovascular death. Sleep Breath. 2010;14(2):131-136.

4. Yaggi HK, Concato J, Kernan WN, et al. Obstructive sleep apnea as a risk factor for stroke and death. N Engl J Med. 2005;353(19):2034-2041.

5. Karger RA, White WA, Park WC, et al. Prevalence of floppy eyelid syndrome in obstructive sleep apnea-hypopnea syndrome. Ophthalmology. 2006;113(9):1669-1674.
6. Bendel RE, Kaplan J, Heckman M, et al. Prevalence of glaucoma in patients with obstructive sleep apnoea - a cross-sectional case-series. Eye (Lond). 2008;22(9):1105-1109.

7. Stein JD, Kim DS, Mundy KM, et al. The association between glaucomatous and other causes of optic neuropathy and sleep apnea. Am J Ophthalmol. 2011;152(6):989-998, e3.

8. West SD, Groves DC, Lipinski HJ, et al. The prevalence of retinopathy in men with Type 2 diabetes and obstructive sleep apnoea. Diabet Med. 2010;27(4):423-430.

9. Glacet-Bernard A, Leroux les Jardins G, Lasry S, et al. Obstructive sleep apnea among patients with retinal vein occlusion. Arch Ophthalmol. 2010;128(12):1533-1538.

10. Cheung CY, Zheng Y, Hsu W, et al. Retinal vascular tortuosity, blood pressure, and cardiovascular risk factors. Ophthalmology. 2011;118(5):812-818.

11. Sasongko MB, Wong TY, Nguyen TT, et al. Retinal vascular tortuosity in persons with diabetes and diabetic retinopathy. Diabetologia. 2011;54(9):2409-2416.

12. Ferrara DC, Koizumi H, Spaide RF. Early bevacizumab treatment of central retinal vein occlusion. Am J Ophthalmol. 2007;144(6): 864-871.

13. Hart WE, Goldbaum M, Cote B, et al. Measurement and classification of retinal vascular tortuosity. Int J Med Inform. 1999;53(2-3):239-252.

14. Young T, Palta M, Dempsey J, et al. The occurrence of sleep-disordered breathing among middle-aged adults. N Engl J Med. 1993;328(17): 1230-1235.

15. Young T, Palta M, Dempsey J, et al. Burden of sleep apnea: rationale, design, and major findings of the Wisconsin Sleep Cohort study. WMJ. 2009;108(5):246-249.

16. Boland LL, Shahar E, Wong TY, et al. Sleep-disordered breathing is not associated with the presence of retinal microvascular abnormalities: the Sleep Heart Health Study. Sleep. 2004;27(3):467-473.

17. Kosseifi S, Bailey B, Price R, et al. The association between obstructive sleep apnea syndrome and microvascular complications in well-controlled diabetic patients. Mil Med. 2010;175(11):913-916.

18. Sho E, Nanjo H, Sho M, et al. Arterial enlargement, tortuosity, and intimal thickening in response to sequential exposure to high and low wall shear stress. J Vasc Surg. 2004;39(3):601-612.

19. Venkataraman ST, Hudson C, Fisher JA, et al. Retinal arteriolar and capillary vascular reactivity in response to isoxic hypercapnia. Exp Eye Res. 2008;87(6):535-542.

20. Urbano F, Roux F, Schindler J, et al. Impaired cerebral autoregulation in obstructive sleep apnea. J Appl Physiol. 2008;105(6):1852-1857.

21. von Känel R, Dimsdale JE. Hemostatic alterations in patients with obstructive sleep apnea and the implications for cardiovascular disease. Chest. 2003;124(5):1956-1967.
Clinical Ophthalmology

\section{Publish your work in this journal}

Clinical Ophthalmology is an international, peer-reviewed journal covering all subspecialties within ophthalmology. Key topics include: Optometry; Visual science; Pharmacology and drug therapy in eye diseases; Basic Sciences; Primary and Secondary eye care; Patient Safety and Quality of Care Improvements. This journal is indexed on Submit your manuscript here: http://www.dovepress.com/clinical-ophthalmology-journal

\section{Dovepress}

PubMed Central and CAS, and is the official journal of The Society of Clinical Ophthalmology (SCO). The manuscript management system is completely online and includes a very quick and fair peer-review system, which is all easy to use. Visit http://www.dovepress.com/ testimonials.php to read real quotes from published authors. 